ARTICLE OPEN

\title{
Activating primary care COPD patients with multi-morbidity through tailored self-management support
}

\author{
Sameera Ansari (iD ${ }^{1 凶}$, Hassan Hosseinzadeh (iD $^{2}$, Sarah Dennis ${ }^{3,4,5,6}$ and Nicholas Zwar ${ }^{1,7}$
}

Given the dearth of COPD self-management interventions that specifically acknowledge multi-morbidity in primary care, we aimed to activate COPD patients through personalised self-management support that recognised the implications of co-morbidities. This single-group experimental study included patients aged 40-84 with a spirometry diagnosis of COPD and at least one co-morbidity. A self-management education programme for COPD in the context of multi-morbidity, based on the Health Belief Model, was tailored and delivered to participants by general practice nurses in face-to-face sessions. At 6 months' follow-up, there was significant improvement in patient activation $(p<0.001)$, COPD-related quality of life $(p=0.012)$, COPD knowledge $(p<0.001)$ and inhaler device technique ( $p=0.001)$, with no significant change in perception of multi-morbidity $(p=0.822)$ or COPD-related multimorbidity (0.084). The programme improved patients' self-efficacy for their COPD as well as overall health behaviour. The findings form an empirical basis for further testing the programme in a large-scale randomised controlled trial.

npj Primary Care Respiratory Medicine (2020)30:12; https://doi.org/10.1038/s41533-020-0171-5

\section{INTRODUCTION}

Chronic obstructive pulmonary disease (COPD) is currently the third leading cause of mortality worldwide, with 3 million people having died of the disease in $2016^{1}$. COPD often occurs in the presence of co-morbidities, which might have concordant or discordant pathophysiology ${ }^{2}$. In Australia, COPD was the fifth leading cause of death in $2015^{3}$, with $91 \%$ of those reported to have COPD experiencing at least one co-morbidity ${ }^{4}$. Co-morbidities contribute to poor health status and higher healthcare utilisation ${ }^{5}$, and aggravate the debilitating nature of COPD ${ }^{6}$.

Since the majority of the Australian population visit their general practitioner (GP) more than once a year ${ }^{7}$, general practice offers the continuity of care needed for interventions aimed at enhancing patients' self-management of chronic disease. With practice nurses (PNs) increasingly contributing to chronic disease management, nurse-delivered self-management support for coordinated care of patients with COPD could lead to significant uptake of interventions shown to improve health outcomes such as smoking cessation, pulmonary rehabilitation and influenza vaccination ${ }^{8,9}$. For such interventions to be effective, patients need to be involved in active discussion that would enhance their self-efficacy for management of COPD ${ }^{10}$.

A qualitative study by the authors found that people with earlystage COPD have difficulty recognising the importance of the condition and its long-term implications and COPD may be given low priority compared to their other co-morbidities ${ }^{11}$. While selfmanagement interventions for patients with COPD can improve health-related quality of life and reduce respiratory-related hospital admissions ${ }^{12}$, most prior studies have focussed on COPD alone and only included patients with moderate or severe disease ${ }^{13-16}$. A meta-analysis of seven studies of communitybased, self-management interventions among primary care COPD patients found no between-group difference in health-related quality of life at final follow-up ${ }^{17}$. There is evidence that a multi- faceted approach is needed to bring about change in health behaviour among those with COPD and providing them with only disease knowledge is not enough ${ }^{18}$. Due to the heterogeneous nature of the disease, effective self-management for COPD needs personalised education and active guidance ${ }^{19}$. Given that most patients with chronic respiratory disease have other chronic conditions ${ }^{20}$, it is essential to include strategies for coping with multi-morbidity in COPD self-management interventions ${ }^{11,21}$.

To date, no research study has provided a behaviour change theory-based, tailored, self-management support for COPD in the context of multi-morbidity, particularly in the primary care setting. The Activating Primary Care COPD Patients with Multimorbidity (APCOM) study aimed to activate patients in regard to their COPD while acknowledging and tailoring support to recognise the implications existing of co-morbidities. It was hypothesised that at 6 months' follow-up after a tailored, selfmanagement education programme, participating patients would have: (i) better activation in terms of their COPD-related health behaviour, (ii) improved knowledge and selfmanagement capacity of COPD, and (iii) increased self-efficacy in terms of their overall health behaviour.

\section{RESULTS}

Recruitment

Among 61 practices invited to participate, 13 (21\%) consented to take part, 17 (28\%) did not respond and 31 (51\%) declined. One practice withdrew due to lack of potential patients after the PN conducted a medical record search. A total of 226 patients were invited to participate in the study (Fig. 1), of whom 50 eventually participated in the study. Each PN managed between two and seven patients during the study.

\footnotetext{
${ }^{1}$ School of Public Health and Community Medicine, UNSW Sydney, Sydney, NSW, Australia. ${ }^{2}$ School of Health and Society, University of Wollongong Australia, Wollongong, NSW, Australia. ${ }^{3}$ Sydney School of Health Sciences, Faculty of Medicine and Health, The University of Sydney, Sydney, NSW, Australia. ${ }^{4}$ Ingham Institute for Applied Medical Research, Sydney, NSW, Australia. ${ }^{5}$ South Western Sydney Local Health District, Sydney, NSW, Australia. ${ }^{6}$ Centre for Primary Health Care and Equity, UNSW Sydney, Sydney, NSW, Australia. ${ }^{7}$ Faculty of Health Sciences and Medicine, Bond University, Gold Coast, QLD, Australia. ${ }^{凶}$ email: sameera.ansari@unsw.edu.au
} 


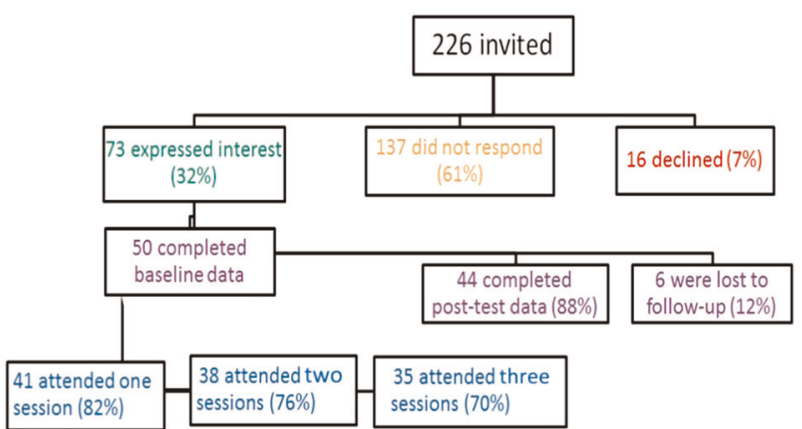

Fig. 1 Recruitment of patient participants and uptake of the programme. It shows the response rate of patient participants and rate of completion of the APCOM programme.

\begin{tabular}{|c|c|}
\hline Characteristic & $N=50$ \\
\hline \multirow[t]{2}{*}{ Sex } & Male: 25 (50\%) \\
\hline & Female: 25 (50\%) \\
\hline Age-Mean years (SD) & $69.22( \pm 8.43)$ \\
\hline \multirow[t]{4}{*}{ Ethnicity } & Caucasian 44 (88\%) \\
\hline & Asian 3 (6\%) \\
\hline & Arabic 2 (4\%) \\
\hline & Other $1(2 \%)$ \\
\hline \multirow[t]{5}{*}{ Level of education } & University 16 (32\%) \\
\hline & Some high school 15 (30\%) \\
\hline & HSC 15 (30\%) \\
\hline & TAFE $3(6 \%)$ \\
\hline & Primary school 1 (2\%) \\
\hline \multirow[t]{4}{*}{ Employment status } & Retired 38 (76\%) \\
\hline & Employed 7 (14\%) \\
\hline & Unemployed 4 (8\%) \\
\hline & Carer $1(2 \%)$ \\
\hline \multirow[t]{2}{*}{ Living arrangement } & With someone 35 (70\%) \\
\hline & Alone 15 (30\%) \\
\hline BMI (SD) & $28.99( \pm 6.19)$ \\
\hline \multirow[t]{3}{*}{ Smoking status } & Ex-smoker 31 (62\%) \\
\hline & Current smoker 12 (24\%) \\
\hline & Never smoked 7 (14\%) \\
\hline \multirow{2}{*}{$\begin{array}{l}\text { Prior experience of pulmonary } \\
\text { rehabilitation }\end{array}$} & Yes $8(16 \%)$ \\
\hline & No 42 (84\%) \\
\hline Outcome measures & Mean (SD) \\
\hline PAM 13 & $57.68( \pm 10.83)$ \\
\hline COPD-Q & $7.24( \pm 2.12)$ \\
\hline CAT & $19.22( \pm 6.95)$ \\
\hline MULTIPleS & $25.3( \pm 14.07)$ \\
\hline COPD-MULTIPleS & $11.76( \pm 6.64)$ \\
\hline Correct inhaler device technique $(n=36)$ & $6(16.67 \%)$ \\
\hline
\end{tabular}

Patient characteristics

Of 50 patients at baseline, 44 completed 6 months' follow-up. Their baseline characteristics are summarised in Table 1. There were a total 90 co-morbidities among the 50 patients at baseline. Hypertension (56\%), hyperlipidaemia (48\%), gastro-oesophageal reflux disease $(40 \%)$, ischaemic heart disease $(38 \%)$, depression (30\%), asthma (28\%), diabetes (28\%), osteoarthritis (24\%), osteoporosis (24\%) and anxiety (22\%) were the ten most recurring co-morbidities.

Change in the study's outcome measures

As seen in Table 2, there was a significant increase $(<0.001)$ of 7.16 points in the Patient Activation Measure 13 (PAM 13), the primary outcome measure. There was also a significant increase $(<0.001)$ of 1.75 in the patients' knowledge of COPD, measured by the COPD Knowledge Questionnaire (COPD-Q), at 6 months' follow-up. The patients' COPD-related quality of life was also significantly improved (0.012), with a change of 2.45 points in their COPD Assessment Test (CAT) score.

The change of 0.44 in patients' perception of the burden of their multi-morbidity, measured by the Multimorbidity Illness Perceptions Scale (MULTIPleS), was not significant $(p=0.822)$, nor was the difference of 1.76 in perception of their COPD in the context of multi-morbidity $(p=0.084)$. There was significant improvement in inhaler device technique $(p=0.001)$, with $50 \%$ more patients having proper inhaler technique at 6 months' follow-up.

Table 3 details the change in outcome measures for a subset of 35 patients who attended all three sessions of the programme. They also had a significant improvement in activation $(p<0.001)$, COPD knowledge $(p<0.001)$, COPD-related quality of life $(0.024)$ and inhaler device technique (0.002). There was no significant change in their perception of multi-morbidity (0.907) or COPDrelated multi-morbidity $(0.237)$.

\section{DISCUSSION}

Our findings indicated that the self-management education programme led to significant improvement in patient activation, COPD knowledge, COPD-related quality of life and inhaler device technique. These changes supported the three hypotheses postulated by the study and also support the idea that a theorybased, tailored self-management programme that recognises and responds to the presence of existing co-morbidities may be more effective than current approaches.

There was a statistically significant increase in the patients' level of activation in terms of their overall health behaviour, as measured by the PAM $13^{22}$. Even though the mean post-test score of the PAM remained in Stage 3 at 6 months' follow-up, the increase of 7.16 points was meaningful as a minimum increase of four points following an intervention suggests a clinical improvement in self-efficacy ${ }^{23}$. This increase in the PAM indicated that, on an average, the patients were in control of their own healthrelated behaviour but needed motivation to adopt and maintain new behaviours ${ }^{24}$.

Prior interventional studies for chronic disease have had similar outcomes in terms of patient activation. A home-based educational intervention for native Americans with type 2 diabetes by Shah et al. ${ }^{25}$ found a significant increase in patient activation. Another self-management intervention for diabetic patients in Norwegian primary care demonstrated a persistent increase in their level of activation ${ }^{26}$. In their secondary analysis of a randomised trial of self-care for patients with depression and comorbidities, McCusker et al. ${ }^{27}$ found that activation significantly improved in both coached and non-coached groups at 6 months' follow-up. Another self-management intervention for COPD in the UK by Turner et al. ${ }^{23}$ demonstrated a significant improvement in paired measures of patient activation.

There was significantly improved COPD-related quality of life following the APCOM programme, as assessed by the CAT ${ }^{28}$. This is in contrast to a previous general practice-based study ${ }^{29}$, and to the findings of the systematic review by Jolly et al. ${ }^{17}$, which found no improvement in health-related quality of life as assessed by the St George's Respiratory Questionnaire. In a trial of selfmanagement for COPD by Bischoff et al. $^{30}$, comprehensive, 
Table 2. Paired comparison of outcome measures of participants who completed 6 months' follow-up.

\begin{tabular}{|c|c|c|c|}
\hline $\mathrm{CAT}^{\mathrm{a}}(n=42)$ & $20( \pm 7)$ & $17.55( \pm 7.7)$ & 0.012 \\
\hline MULTIPleS $^{\mathrm{a}}(n=43)$ & $25.58( \pm 14.15)$ & $26.02( \pm 11.43)$ & 0.822 \\
\hline COPD-MULTIPIeS ${ }^{\mathrm{a}}(n=42)$ & $12.48( \pm 6.8)$ & $10.71( \pm 6.05)$ & 0.084 \\
\hline
\end{tabular}

Table 3. Paired comparison of outcome measures of subset who completed the programme.

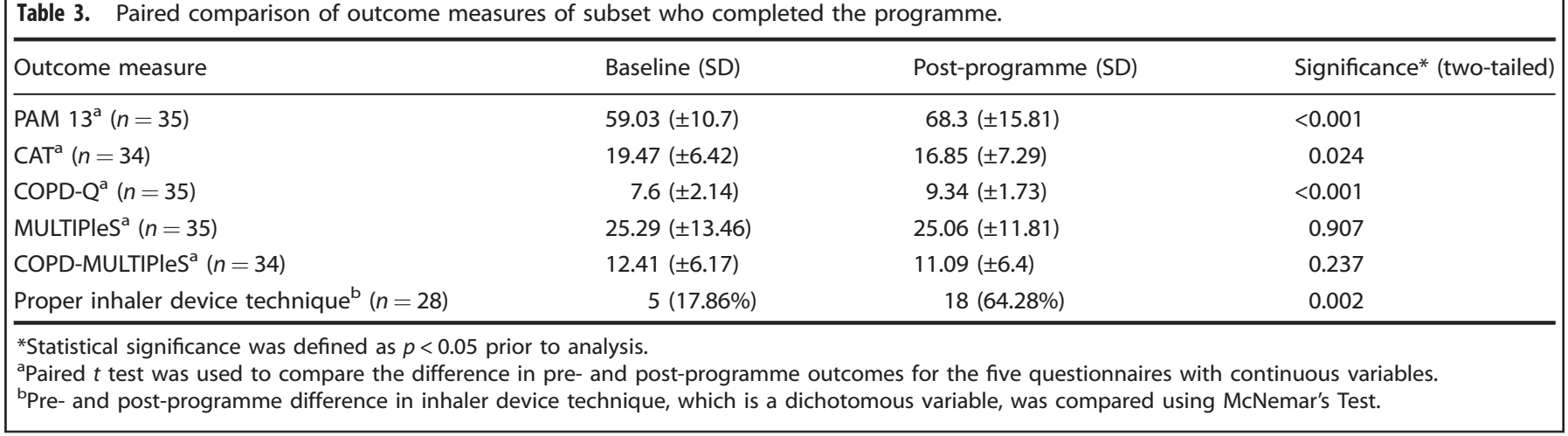

tailored PN-delivered education and routine monitoring did not show better quality of life or self-efficacy. This could be attributed to the single disease-focussed nature of their intervention. Some prior self-management interventions for COPD have yielded positive outcomes. Another PN-led self-management intervention for patients with COPD in the UK showed a significant improvement in their CAT scores ${ }^{31}$, which are in line with our study's findings. A pilot trial of self-management support for primary care patients with COPD in the UK saw an improvement in their health-related quality of life at 6 months' follow-up, but this study only recruited patients with moderate-to-severe disease ${ }^{14}$.

There was a significant improvement in the patients' knowledge of their COPD following the APCOM programme, which was measured by the COPD- $\mathrm{Q}^{32}$. Increase in patients' COPD knowledge was also observed following an interdisciplinary programme to enhance management of asthma and COPD in primary care ${ }^{33}$. There was no significant change in the patients' perception of their multi-morbidity, or COPD in the context of multi-morbidity, following the programme. This might be because the patients were already aware of the burden imposed by their various chronic conditions. A British survey that explored factors predicting self-management behaviour ${ }^{34}$ found that patients' experience of multi-morbidity, based on the MULTIPleS ${ }^{35}$ scale, was not a critical predictor of self-management; self-management behaviour was predicted by illness perceptions around the consequences of individual conditions.

The patients' inhaler device technique ${ }^{36}$ significantly improved following the programme. It is interesting that no prior selfmanagement interventions for COPD in primary care seemed to have assessed inhaler technique. This might be due to the lack of a standard method for assessing inhaler technique for different types of devices, which imposes a barrier towards its accurate assessment $^{37}$.
To the best of our knowledge, the innovative APCOM programme was a first to provide tailored, self-management support for primary care patients with COPD in the context of multi-morbidity. Unlike other self-management programmes for COPD that used existing interventions and mainly focussed on physical outcomes ${ }^{30,38}$, our multi-faceted programme was newly developed and incorporated a biopsychosocial perspective. This entailed looking at biomedical as well as psychological aspects ${ }^{39}$, and being considerate of patients' preferences, needs and values, which are essential for providing self-management support for chronic disease ${ }^{40}$. As opposed to other self-management interventions for COPD in primary care which had a single disease focus $^{13,14}$, the APCOM programme considered the implications of patients' co-morbidities towards their COPD. Patients were included irrespective of the severity of their COPD, unlike a British practice nurse-led, self-management trial of telephone health coaching, which only included patients with mild symptoms of the disease ${ }^{41}$.

All participating general practices and PNs remained in the study, with $12 \%$ of the patients being lost to follow-up, which was lower than the anticipated dropout rate of $20 \%{ }^{42}$. This shows feasibility of recruitment and retention of practices for a largescale trial. The response rate of $88 \%$ at 6 months' follow-up in our study was higher than a group self-management initiative for COPD, which also used PAM 13 as the primary outcome measure but had a response rate of $47 \%{ }^{23}$. This is further indication of the effectiveness of personalised self-management support for empowering patients with COPD. The sample size of our study was adequate for test-retest reliability of the outcome measures $^{43}$. Although a confidence interval of about $80 \%$ is deemed sufficient for analysis of data from pilot studies ${ }^{44}$, the confidence interval for analysis of the study's endpoints was set to $95 \%$. 
A major limitation of our study was the lack of a control group, which makes it difficult to ascertain whether post-programme improvement in the outcome measures were solely due to the programme ${ }^{43}$. Inclusion of a control group would have required 30 more patient participants $(N=80)$, with 40 in each group to estimate test-retest reliability ${ }^{42}$. This would have required at least six more PNs and practices, further extending the time for practice recruitment, which was about 8 months for our study. Given that the main purpose of this study was to pilot test the effect of a complex intervention in a real-world clinical setting, it was not essential to include a control group ${ }^{45}$.

Given the positive finding from our pilot study, a large-scale randomised controlled trial based on findings of the APCOM study is recommended as the next step for research. Such a study needs to be adequately powered to show a difference in health-related quality of life and health service utilisation. Interventional studies are needed for Indigenous patients with COPD and co-morbidities, in view of greater incidence of the disease in Indigenous Australians. Initiatives to deliver such tailored, self-management support for other chronic conditions need to be implemented in routine general practice, irrespective of who delivers the programme. Patients should be educated regarding the correct inhaler device technique upon initial prescription, and their technique should be assessed regularly during GP and PN consultations.

In conclusion, the tailored, self-management education programme piloted in our study led to improvement in patients' selfefficacy in terms of their COPD and overall health behaviour. There was significant increase in patient activation, COPD knowledge, COPD-related quality of life and inhaler device technique at 6 months' follow-up. There was no significant difference in patients' perception of their multi-morbidity or COPD-related multi-morbidity. The study echoes the growing need for personalised self-management support for COPD in the context of multi-morbidity at the primary care level. The findings are an empirical basis for testing the innovative programme as a future large-scale randomised controlled trial.

\section{METHODS}

The APCOM study was a single-group, pre- and post-intervention study conducted in participating general practices across metropolitan Sydney from mid-2015 to December 2016. The study comprised development, piloting and evaluation of an innovative self-management education programme for COPD in the context of multi-morbidity. The study protocol and components of the programme have been described in detail elsewhere ${ }^{46}$. Ethics approval was obtained from the Human Research Ethics Committee of UNSW Sydney (HREC14139).

\section{Participant eligibility}

General practices were eligible to participate if they maintained an electronic database of medical records and employed one or more PN. Patients were included if they: (i) were aged between 40 and 84 years, (ii) had a record of spirometry diagnosis of COPD in their practice notes and (iii) had at least one other co-existing chronic condition or co-morbidity. Patients were excluded if they had cognitive impairment or were unable to understand sufficient English to complete the study questionnaires and follow the intervention.

\section{Recruitment}

General practices were invited to participate from the Practice Based Research Network at UNSW Sydney, a group of practices that had previously participated in research studies conducted by N.Z. and S.D., or expressed an interest in research participation. An information sheet about the study was faxed to potential general practices. Practices that did not respond within 2 weeks of the initial invitation were followed up twice by telephone and/or email. S.A. visited practices that expressed an interest in taking part in the study and met with the PNs and GPs, briefed them about the study and obtained their written informed consent.
The PNs were provided with instructions on how to search for eligible patients in the practice records based on the inclusion criteria. All potentially eligible patients were sent an invitation letter, including a brief description of the study. Non-responders were reminded by the PNs via telephone after 2 weeks. Each PN was asked to recruit five patients for the study since this number seemed feasible to all.

\section{Intervention}

The details of the intervention have been included in the published study protocol ${ }^{46}$. In brief, participating PNs attended 1-day workshops facilitated by the authors and were trained to deliver the self-management education programme. The PNs were trained to conduct a patient assessment using a template based on the Health Belief Model ${ }^{47}$. This assessment identified the patients' health priorities and was used by the PNs to facilitate development of tailored strategies for the patients to support self-management of COPD in the context of multi-morbidity. One of the authors (S.A.) provided research support to the PNs during the 6-week programme, which comprised three face-to-face sessions spaced 2 weeks apart.

During the first PN-patient session, individual patient needs were assessed and the intervention tailored accordingly. The PNs were asked to use Motivational Interviewing ${ }^{48}$ to address barriers faced by the patients in managing their COPD in the face of co-morbidities. Health information and referrals to healthcare providers were provided as necessary. Following the last session, the PNs followed up with the patients via a monthly phone call for 5 months.

\section{Outcome measures}

The primary outcome was the Patient Activation Measure (PAM 13) 22. Secondary outcomes were COPD Assessment Test (CAT) ${ }^{28}$, COPD Knowledge Questionnaire (COPD-Q) $)^{32}$, Multimorbidity Illness Perceptions Scale (MULTIPleS $)^{35}$, COPD-specific version of MULTIPleS and inhaler device technique ${ }^{36}$. The first hypothesis of the study was addressed by the PAM 13, COPD-Q, CAT, COPD-MULTIPleS and inhaler device technique, the second hypothesis was addressed by the COPD-Q, CAT and inhaler device technique, and the third hypothesis was addressed by the PAM 13 and MULTIPleS.

\section{Reporting summary}

Further information on research design is available in the Nature Research Reporting Summary linked to this article.

\section{DATA AVAILABILITY}

The datasets generated during and/or analysed during the current study are available from the corresponding author on reasonable request.

Received: 28 April 2019; Accepted: 5 March 2020;

Published online: 03 April 2020

\section{REFERENCES}

1. World Health Organization. The top 10 causes of death. https://www.who.int/ news-room/fact-sheets/detail/the-top-10-causes-of-death (2018).

2. Brown, J. P. \& Martinez, C. H. Chronic obstructive pulmonary disease comorbidities. Curr. Opin. Pulm. Med. 22, 113-118 (2016).

3. Australian Institute of Health and Welfare. COPD (chronic obstructive pulmonary disease) snapshot. https://www.aihw.gov.au/reports/chronic-respiratory-conditions/ copd/contents/copd (2018).

4. Australian Institute of Health and Welfare. COPD, associated comorbidities and risk factors. https://www.aihw.gov.au/reports/chronic-respiratory-conditions/ copd-associated-comorbidities-risk-factors/contents/about-copd-andassociated-comorbidities (2016).

5. Burgel, P. R. et al. Impact of comorbidities on COPD-specific health-related quality of life. Respir. Med. 107, 233-241 (2013).

6. Franssen, F. M. E. The physical, mental, and social impact of COPD in a population-based sample: results from the Longitudinal Aging Study Amsterdam. npj Prim. Care Respir. Med. 28, 30 (2018).

7. Harrison, C., Henderson, J., Miller, G. \& Britt, H. The prevalence of diagnosed chronic conditions and multimorbidity in Australia: a method for estimating population prevalence from general practice patient encounter data. PLOS ONE 12, e0172935 (2017) 
8. Zwar, N. et al. A cluster randomised controlled trial of nurse and GP partnership for care of chronic obstructive pulmonary disease. BMC Pulm. Med. 8, 8 (2008).

9. Zwar, N. A. et al. Early intervention for chronic obstructive pulmonary disease by practice nurse and GP teams: a cluster randomized trial. Fam. Pract. 33, 663-670 (2016).

10. Verbrugge, R., de Boer, F. \& Georges, J. Strategies used by respiratory nurses to stimulate self-management in patients with COPD. J. Clin. Nurs. 22, 2787-2799 (2013).

11. Ansari, S., Hosseinzadeh, H., Dennis, S. \& Zwar, N. Patients' perspectives on the impact of a new COPD diagnosis in the face of multimorbidity: a qualitative study. npj Prim. Care Respir. Med. 24, 14030 (2014).

12. Bourbeau, J., Nault, D. \& Dang-Tan, T. Self-management and behaviour modification in COPD. Patient Educ. Couns. 52, 271-277 (2004).

13. Taylor, S. J. C. et al. Self-management support for moderate-to-severe chronic obstructive pulmonary disease: a pilot randomised controlled trial. Br. J. Gen. Pract. 62, e687-e695 (2012).

14. Walters, J. et al. Effects of telephone health mentoring in community-recruited chronic obstructive pulmonary disease on self-management capacity, quality of life and psychological morbidity: a randomised controlled trial. BMJ Open 3, e003097 (2013)

15. Jonsdottir, $H$. et al. Effectiveness of a partnership-based self-management programme for patients with mild and moderate chronic obstructive pulmonary disease: a pragmatic randomized controlled trial. J. Adv. Nurs. 71, 2634-2649 (2015).

16. Jonkman, N. H. et al. Do self-management interventions in COPD patients work and which patients benefit most? An individual patient data meta-analysis. Int. J. COPD 11, 2063-2074 (2016).

17. Jolly, K. et al. Systematic review of the effectiveness of community-based selfmanagement interventions among primary care COPD Patients. npj Prim. Care Respir. Med. 28, 44 (2018).

18. Effing, T., van der Palen, J. \& Frith, P. Education in COPD self-management: only part of the game. Respirology 19, 151-152 (2014).

19. Kruis, A. L., van Schayck, O. C. P., in't Veen, J. C. C. M., van der Molen, T. \& Chavannes, N. H. Successful patient self-management of COPD requires hands-on guidance. Lancet Respir. Med. 1, 670-672 (2013). November.

20. O'Kelly, S., Smith, S. M., Lane, S., Teljeur, C. \& O'Dowd, T. Chronic respiratory disease and multimorbidity: prevalence and impact in a general practice setting. Respir. Med. 105, 236-242 (2011).

21. Ansari, S. Understanding the impact of chronic obstructive pulmonary disease and intervening to improve self-management in the context of multi-morbidity. Int. J. Integr. Care 18, 7 (2018).

22. Hibbard, J. H., Mahoney, E. R., Stockard, J. \& Tusler, M. Development and testing of a short form of the patient activation measure. Health Serv. Res. 40, 1918-1930 (2005)

23. Turner, A. P., Anderson, J. K., Wallace, L. M. \& Kennedy-Williams, P. Evaluation of a self-management programme for patients with chronic obstructive pulmonary disease. Chronic Respir. Dis. 11, 163-172 (2014).

24. Chubak, J. et al. Predictors of 1-year change in patient activation in older adults with diabetes mellitus and heart disease. J. Am. Geriatr. Soc. 60, 1316-1321 (2012).

25. Shah, V. O. et al. A home-based educational intervention improves patient activation measures and diabetes health indicators among Zuni Indians. PLOS ONE 10, e0125820 (2015).

26. Fløde, M., Iversen, M. M., Aarflot, M. \& Haltbakk, J. Lasting impact of an implemented self-management programme for people with type 2 diabetes referred from primary care: a one-group, before-after design. Scand. J. Caring Sci. 31, 789-795 (2017)

27. McCusker, J. et al. Activation and self-efficacy in a randomized trial of a depression self-care intervention. Health Educ. Behav. 43, 716-725 (2016)

28. Papaioannou, M. et al. COPD Assessment Test: a simple tool to evaluate disease severity and response to treatment. COPD 11, 489-495 (2014).

29. McGeoch, G. R. B. et al. Self-management plans in the primary care of patients with chronic obstructive pulmonary disease. Respirology 11, 611-618 (2006).

30. Bischoff EWMA et al. Comprehensive self management and routine monitoring in chronic obstructive pulmonary disease patients in general practice: randomised controlled trial. BMJ 345, e7642 (2012).

31. Billington, J., Coster, S., Murrells, T. \& Norman, I. Evaluation of a nurse-led educational telephone intervention to support self-management of patients with chronic obstructive pulmonary disease: a randomized feasibility study. COPD 12, 395-403 (2015).

32. Ray, S. M., Helmer, R. S., Stevens, A. B., Franks, A. S. \& Wallace, L. S. Clinical utility of the chronic obstructive pulmonary disease knowledge questionnaire. Fam. Med. 45, 197-200 (2013)
33. Wheeler, J. S., Ray, S. M., Helmer, R. S., Stevens, A. B. \& Franks, A. S. Development and implementation of an interdisciplinary pulmonary care clinic within an existing clinic program. Am. J. Health-Syst. Pharm. 72, 1844-1847 (2015).

34. Kenning, C. et al. Does patient experience of multimorbidity predict selfmanagement and health outcomes in a prospective study in primary care? Fam. Pract. 32, 311-316 (2015).

35. Gibbons, C. J. et al. Development of a Multimorbidity Illness Perceptions Scale (MULTIPleS). PLoS ONE 8, e81852 (2013).

36. Armour, C. L. et al. Using the community pharmacy to identify patients at risk of poor asthma control and factors which contribute to this poor control. J. Asthma 48, 914-922 (2011).

37. Molimard, M. et al. Chronic obstructive pulmonary disease exacerbation and inhaler device handling: real-life assessment of 2935 patients. Eur. Respir. J. 49, 1601794 (2017).

38. Ashmore, J. et al. Chronic obstructive pulmonary disease self-management activation research trial (COPD-SMART): design and methods. Contemp. Clin. Trials 35, 77-86 (2013).

39. van Dijk-de Vries, A., van Bokhoven, M. A., Terluin, B., van der Weijden, T. \& van Eijk, J. T. M. Integrating nurse-led Self-Management Support (SMS) in routine primary care: design of a hybrid effectiveness-implementation study among type 2 diabetes patients with problems of daily functioning and emotional distress: a study protocol. BMC Fam. Pract. 14, 77 (2013).

40. Kaptein, A. A., Fischer, M. J. \& Scharloo, M. Self-management in patients with COPD: theoretical context, content, outcomes, and integration into clinical care. Int. J. COPD 9, 907-917 (2014).

41. Sidhu, M. S. et al. Patient self-management in primary care patients with mild COPD-protocol of a randomised controlled trial of telephone health coaching BMC Pulm. Med. 15, 16 (2015).

42. Armijo-Olivo, S., Warren, S. \& Magee, D. Intention to treat analysis, compliance, drop-outs and how to deal with missing data in clinical research: a review. Phys. Ther. Rev. 14, 1 (2009).

43. Hertzog, M. A. Considerations in determining sample size for pilot studies. Res. Nurs. Health 31, 180-191 (2008).

44. Lee, E. C., Whitehead, A. L., Jacques, R. M. \& Julious, S. A. The statistical interpretation of pilot trials: should significance thresholds be reconsidered? BMC Med. Res. Methodol. 14, 41 (2014).

45. Leon, A. C., Davis, L. L. \& Kraemer, H. C. The role and interpretation of pilot studies in clinical research. J. Psychiatr. Res. 45, 626-629 (2011).

46. Ansari, S., Hosseinzadeh, H., Dennis, S. \& Zwar, N. Activating primary care COPD patients with multi-morbidity (APCOM) pilot project: study protocol. npj Prim. Care Respir. Med. 27, 12 (2017).

47. Hayden, J. A. Introduction to Health Behavior Theory 1st edn, Ch. 4, 31-44 (Jones and Bartlett Publishers, 2009).

48. Hettema, J., Steele, J. \& Miller, W. R. Motivational interviewing. Annu. Rev. Clin. Psychol. 1, 91-111 (2005).

\section{ACKNOWLEDGEMENTS}

The APCOM pilot study was funded by GlaxoSmithKline Australia (Study number 204950) and recognised by the Australian National Health and Medical Research Council through a Ph.D. scholarship awarded to S.A. (GNT1093032). The authors are grateful to all the general practices, PNs, GPs and patients who participated in this study. We thank Ms. An Tran for her assistance with practice recruitment, data entry and organising the first training workshop.

\section{AUTHOR CONTRIBUTIONS}

All authors conceptualised the study and were involved in workshop delivery. S.A. recruited participants, developed the intervention resources, organised the training workshops, coordinated the study, collected and analysed the data, drafted the manuscript and coordinated its revision. H.H., S.D. and N.Z. critically appraised the manuscript, contributed to its revision and approved the final draft.

\section{COMPETING INTERESTS}

The authors declare no competing interests.

\section{ADDITIONAL INFORMATION}

Supplementary information is available for this paper at https://doi.org/10.1038/ s41533-020-0171-5.

Correspondence and requests for materials should be addressed to S.A. 
Reprints and permission information is available at http://www.nature.com/ reprints

Publisher's note Springer Nature remains neutral with regard to jurisdictional claims in published maps and institutional affiliations.

Open Access This article is licensed under a Creative Commons Attribution 4.0 International License, which permits use, sharing, adaptation, distribution and reproduction in any medium or format, as long as you give appropriate credit to the original author(s) and the source, provide a link to the Creative Commons license, and indicate if changes were made. The images or other third party material in this article are included in the article's Creative Commons license, unless indicated otherwise in a credit line to the material. If material is not included in the article's Creative Commons license and your intended use is not permitted by statutory regulation or exceeds the permitted use, you will need to obtain permission directly from the copyright holder. To view a copy of this license, visit http://creativecommons. org/licenses/by/4.0/.

(c) The Author(s) 2020 